\title{
Road Bank Angle Estimation for Three Wheel Tilting Vehicle Using Multi Model Estimator
}

\author{
Kicheol Jeong ${ }^{1}$, Seibum Choi ${ }^{1, a}$ \\ ${ }^{1} K A I S T$, Department of Mechanical Engineering, 34141 Deajeon, Republic of Korea
}

\begin{abstract}
In recent years, the need for micro-mobility, especially three-wheel vehicles, is increasing to address pollution and traffic congestion problems. With regard to the development of tilting vehicles, the precise tilt angle is important information in the tilting mechanism. Since the road environment affects the vehicle tilt angle, the road bank and hill angle have to be estimated to optimize the tilt control system. Furthermore, a new tilt mechanism is required due to the energy consumption of the previous active tilting mechanism. This paper introduces the road state observer. In this paper, the observer that combines a kinematic model with a dynamic model of a three wheel vehicle is proposed. The dynamic model has four states, including lateral velocity, yaw rate, tilt angle, and tilt angle ratio. Similarly, kinematic model has two states, including roll and pitch angles. It is assumed that the data set received from the six-dimensional inertial measurement unit including the vehicle acceleration and angular velocity of all axes is available. To verify the proposed algorithm, simulation verification using Carsim ADAMS and Matlab\&Simulink is performed and a discussion of the result is provided. In addition, this paper proposes a semi-active tilt system.
\end{abstract}

\section{Introduction}

Recently, conventional transportation systems cause many problems. From an ecological point of view, interest in air pollution and depletion of fossil fuel problems has increased. Furthermore, in modern cities, traffic volume and parking lot shortages are becoming more serious

In this situation, narrow and short micro-mobility, especially three wheel vehicle, is interested in solving this phenomenon $[1,2]$. This small vehicle operates as an electric power train system, so there is no exhaust, parking and traffic congestion. However, because of the body shape of this vehicle, it has a high center of gravity. This physical property makes this vehicle vulnerable to rollover. To overcome this drawback, a three wheel vehicle must include a lean system that compensates for the roll stability of the mobility during cornering maneuver [3-5].

It is important to determine the exact tilt angle to control the vehicle tilt system. Because road bank and hill angles have an impact on the vehicle tilt angle, the road characteristics have to be estimated to optimize the tilting control system. In previous studies on road angles, S. Kidane directly calculated road bank angles using vehicle dynamic models [6]. However, this method has a limitation that the logic is unstable when the vehicle has a small tilt angle.

In this paper, the road state observer combining the three wheel vehicle dynamic model and the kinematic model is proposed. The dynamic model has four states, including lateral velocity, yaw rate, tilt angle and tilt angle rate. Likewise, the kinematic model has two states including roll and pitch angle. It is assumed that a set of data received from a six dimensional-inertial measurement unit (6D-IMU) that provides the vehicle linear acceleration and angular rates of all axes is available.

The proposed observer in this paper is organized as follows. First, the pseudo-measurement is generated from a multi-model based pseudo-angle generator. This generator is based on the three wheel vehicle dynamic model and a Kalman filter based on type 1 Euler angle kinematic model. Depending on the condition of the vehicle state, the generator generates an accurate pseudomeasurement that is used to estimate the vehicle roll and pitch angle. Next, the vehicle roll and pitch angle are obtained with a kinematic model-based linear observer. This observer is based on a kinematic model and uses pseudo measurement as the sensor measurement. Finally, the bank angle is obtained from the road bank angle calculation process.

This paper is organized as follows. Section 2.1 contains the demonstration of the three wheel vehicle dynamics. Section 2.2 explains the pseudo-measurement generator. Section 2.3 explains linear estimator, section 2.4 explains nonlinear estimator and section 2.5 describe the bank angle calculation scheme. Next, section 3 shows the simulation verification result of the observer. Finally, section 4 deals with the conclusion of this paper.

\footnotetext{
a Corresponding author: sbchoi@kaist.ac.kr
} 


\section{Road bank estimator}

\subsection{Three wheel vehicle dynamic model}

\subsubsection{Vehicle dynamic model using gravity transformation}

To illustrate the tilt behavior of a vehicle, a specialized three wheel vehicle dynamic model is required. In particular, the road angle affects the vehicle tilt angle. Therefore, when deriving a vehicle dynamic model, the angle of the road should be considered.



Figure 1. Three wheel vehicle free body diagram

As shown in Figure 1, the road angle has a significant impact on vehicle tilt dynamics. According to this free body diagram, the gravity coordinate transformation matrix can be obtained.

$$
\begin{gathered}
R_{S}=\left[\begin{array}{ccc}
\cos \sigma & 0 & \sin \sigma \\
0 & 1 & 0 \\
-\sin \sigma & 0 & \cos \sigma
\end{array}\right] \\
R_{B}=\left[\begin{array}{ccc}
1 & 0 & 0 \\
0 & \cos \tau & -\sin \tau \\
0 & \sin \tau & \cos \tau
\end{array}\right] \\
G_{T}=R_{B} R_{S} G_{N}=\left[\begin{array}{c}
-g \sin \sigma \\
g \sin \tau \cos \sigma \\
-g \cos \tau \cos \sigma
\end{array}\right]
\end{gathered}
$$

The above matrix is a gravity transformation matrix. Equation (1) is a longitudinal gradient conversion matrix, and equation (2) is a lateral gradient conversion matrix The matrix makes deriving mathematical model process simple. Using this transformation matrix, three-wheel vehicle dynamic model can be obtained as follows.

$$
A=\left[\begin{array}{cccc}
-\frac{\alpha}{m v}\left(2 C_{f}+C_{r}\right) & -\left(v+\frac{\alpha}{m v}\left(2 C_{f} l_{f}-C_{r} l_{r}\right)\right) & \frac{h k}{I_{x x}}-\frac{m h^{2}}{I_{x x}} g & \frac{h \gamma}{I_{x x}} \\
\frac{C_{r} l_{r}-2 C_{f} l_{f}}{v I_{z}} & -\frac{2 C_{f} l_{f}^{2}+C_{r} l_{r}^{2}}{v I_{z}} & 0 & 0 \\
0 & 0 & 0 & 1 \\
h \frac{2 C_{f}+C_{r}}{I_{x x} v} & h \frac{2 l_{f} C_{f}-l_{r} C_{r}}{I_{x x} v} & \frac{m g h}{I_{x x}}-\frac{k}{I_{x x}} & -\frac{\gamma}{I_{x x}}
\end{array}\right]
$$

This is a state space representation model, which includes the lateral velocity, yaw rate, tilt angle, and tilt velocity. In this paper, vehicle tire force is assumed to be a linear tire force. In previous studies like [7], other vehicle tire model is presented, but are generally suitable for high speed conventional four wheel vehicles. This assumption is reasonable because a three wheel tilting vehicle has a relatively low speeds in general.

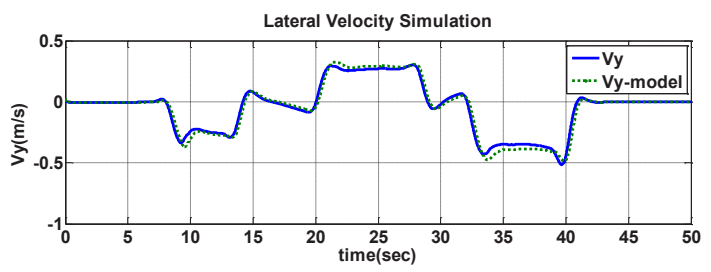

Figure 2. Model verification result

The above figure shows the model verification result. Model and simulation plant outputs for the same steering inputs are similar. Therefore, the model is well defined and can be used to construct estimators.

\subsubsection{Design the desired tilt angle}

In this paper, the lateral acceleration of the vehicle can be obtained from the sensor. However, the sensor signals are contaminated with roadways, vehicle inclination angles, and tilt angles.

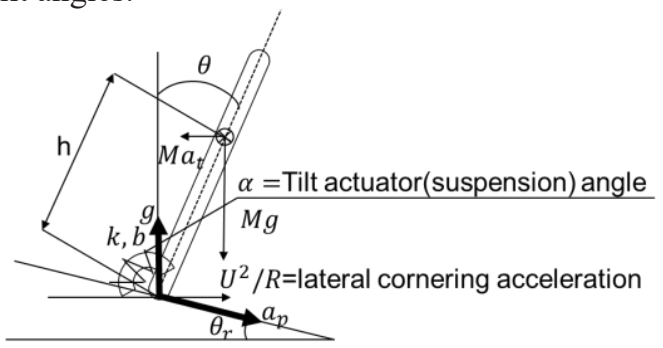

Figure 3. Earth fixed coordinate model

This figure shows the cornering with banked road situation. In this situation, the road bank, turning radius and vehicle velocity have an impact on the vehicle roll dynamics.

$$
\begin{gathered}
I \ddot{\theta}=m g h \sin \theta-m h a_{c}+b\left(\dot{\alpha}+\dot{\theta_{r}}-\dot{\theta}\right) \\
+k\left(\alpha+\theta_{r}-\theta\right) \\
a_{c}=\frac{U_{1}^{2}}{R_{0}} \\
a_{p}=\frac{U^{2}}{R} \cos \theta-g \sin \theta \\
\tan \theta_{d}=\frac{U^{2}}{R g}
\end{gathered}
$$

This equation shows that the road bank angle affects the desired tilt angle. Equation (8) is the equation for the desired tilt angle. According to this equation, lateral acceleration does not affect vehicle movement when the vehicle is tilted to the desired tilt angle.

\subsection{Pseudo measurement generator}




\subsubsection{Dynamic model based pseudo measurement generation}

In this section, a pseudo measure generator is proposed. Since the vehicle roll and pitch angle cannot be measured, the pseudo measure replaces the actual measurement of the design roll and pitch angle observer. In a previous study, Eric Tseng introduced a pseudo measurement based on Euler angles of type 1 [8]. However, this study assumed that the lateral speed of the vehicle was negligible. Unlike other conventional vehicles, the three wheel tilting vehicle has a relatively high lateral velocity because tilt angular velocity is applied to the lateral velocity when the vehicle is tilted. Therefore, this paper introduces the lateral velocity compensation method using Kalman filter. The Kalman filter model is based on a dynamic model of a three-wheeled vehicle, which includes yaw rate, tilt angle and tilt speed. Then the vehicle lateral velocity can be obtained and compensate the kinematic model based pseudo measurement.

$$
\begin{aligned}
& \left\{\begin{array}{c}
\hat{x}_{k}^{-}=F_{k-1} \hat{x}_{k-1}^{+}+G_{k-1} u_{k-1} \\
P_{k}^{-}=F_{k-1} P_{k-1}^{+} F_{k-1}^{T}+Q_{k-1} \\
K_{k}=P_{k}^{-} H_{k}^{T}\left[H_{k} P_{k}^{-} H_{k}^{T}+R_{k}\right]^{-1} \\
\hat{x}_{k}^{+}=\hat{x}_{k}^{-}+K_{k}\left[z_{k}-H_{k} \hat{x}_{k}^{-}\right] \\
P_{k}^{+}=\left(I-K_{k} H_{k}\right) P_{k}^{-}
\end{array}\right. \\
& \theta_{\text {refM }}=\sin ^{-1}\left(\frac{\dot{v}_{\text {wheel }}-a_{x}-r \cdot \hat{v}_{y}}{g}\right) \\
& \phi_{r e f M}=\sin ^{-1}\left(\frac{-\dot{\hat{v}}_{y}+a_{y}-r \cdot v_{\text {wheel }}}{g \cdot \cos \theta_{r e f M}}\right)
\end{aligned}
$$

With this dynamic model-based Kalman filter, relatively accurate pseudo-measurements can be obtained when the vehicle is in a steady state. However, when the vehicle is in a transient state, the accuracy of this dynamic model-based pseudo measurement decreases. Therefore, another pseudo measurement generation algorithm is needed to ensure accuracy in transient situations.

\subsubsection{Kinematic model based pseudo measurement generation}

The pseudo roll and pitch angle can be obtained kinematic model. The kinematic roll and pitch model are expressed as the following:

$$
\begin{gathered}
\phi_{\text {int }}=\int p+(q \sin \phi+r \cos \phi) \tan \theta d t \\
\theta_{\text {int }}=\int q \cos \phi-r \sin \phi d t
\end{gathered}
$$

Then another pseudo measurements based on the kinematic model based Kalman filter can be obtained. This measurements is accurate under transient conditions, but relatively inaccurate when the vehicle is state.
According to equation (9), the high measurement error covariance makes the Kalman filter estimation result dependent on the state model rather than the measurement. By the contrary, the low measurement error covariance makes estimation result dependent on the measurement information. Therefore, in this paper, steady state factor (SF) that adjust the filter measurement error covariance is proposed. This element is a function of the norm of the acceleration. If the vehicle is in transient state, SF is reduced to zero. In the contrary, if the vehicle is stable, SF is increased to one.

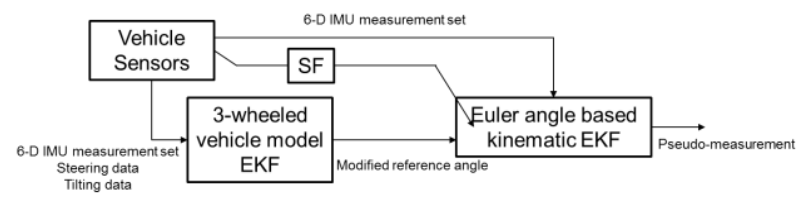

Figure 4. Pseudo-measurement generation scheme

\subsection{Linear roll and pitch angle estimator}

This section, linear vehicle roll and pitch angle estimator is developed. The linear estimator is based on simple kinematic model.

$$
\begin{gathered}
\dot{\phi}=p+(q \sin \phi+r \cos \phi) \tan \theta \\
\dot{\theta}=q \cos \phi-r \sin \phi \\
\Delta \phi=q \cdot \sin \phi \cdot \tan \theta+r \cdot(\cos \phi \cdot \tan \theta-\theta) \\
\Delta \theta=q \cdot(\cos \phi-1)+r \cdot(\phi-\sin \phi)
\end{gathered}
$$

The delta term above is a nonlinear element of equation (14) and (15). Even if the vehicle is leaning from the maximum tilt angle, the magnitude of this nonlinear term is negligible. Therefore, the linear estimator can be designed and the form of the estimator as follows.

$$
\begin{gathered}
{\left[\begin{array}{c}
\dot{\hat{\phi}} \\
\dot{\hat{\theta}}
\end{array}\right]=\left[\begin{array}{cc}
0 & r \\
-r & 0
\end{array}\right] \cdot\left[\begin{array}{l}
\hat{\phi} \\
\hat{\theta}
\end{array}\right]+L \cdot\left[\begin{array}{c}
\phi_{\text {pseudo }}-\hat{\phi} \\
\theta_{\text {pseudo }}-\hat{\theta}
\end{array}\right]} \\
L=\left[\begin{array}{ll}
L_{1} & L_{2} \\
L_{2} & L_{3}
\end{array}\right] \\
{\left[\begin{array}{c}
\dot{\hat{\phi}} \\
\dot{\hat{\theta}}
\end{array}\right]=\left[\begin{array}{cc}
-L_{1} & r-L_{2} \\
-r-L_{2} & -L_{3}
\end{array}\right] \cdot\left[\begin{array}{l}
\hat{\phi} \\
\hat{\theta}
\end{array}\right]+L \cdot\left[\begin{array}{l}
\phi_{\text {pseudo }} \\
\theta_{\text {pseudo }}
\end{array}\right]} \\
\left|\lambda I-A^{*}\right|=\left[\begin{array}{cc}
\lambda+L_{1} & -r+L_{2} \\
+r+L_{2} & \lambda+L_{3}
\end{array}\right] \mid=\left(\lambda+L_{1}\right)\left(\lambda+L_{3}\right)-\left(L_{2}^{2}-r^{2}\right)
\end{gathered}
$$

Observer gain (19) should be selected to stabilize the observer system (21). According to equation (22), if the 
diagonal elements of the observer gain matrix are positive and the other elements are equal to yaw rate, then this observer is stable.

\subsection{Nonlinear roll and pitch angle estimator}

Therewith, in this paper, nonlinear estimation logic also proposed. Nonlinear terms are negligible, but large tilt angles make nonlinear effects larger. The nonlinear estimator can be established as the following:

$$
\begin{gathered}
\dot{\hat{\phi}}=p+(q \sin \hat{\phi}+r \cos \hat{\phi}) \tan \hat{\theta}+k_{1} \operatorname{sgn}\left(\phi_{p s e u d o}-\hat{\phi}\right) \\
\dot{\hat{\theta}}=q \cos \hat{\phi}-r \sin \hat{\phi}+k_{2} \operatorname{sgn}\left(\theta_{\text {pseudo }}-\hat{\theta}\right) \\
\dot{s}_{1}=\dot{\varepsilon}_{\phi}=\Delta f_{\phi}-k_{1} \operatorname{sgn}\left(s_{1}\right) \\
\dot{s}_{2}=\Delta f_{\theta}-k_{2} \operatorname{sgn}\left(s_{2}\right)
\end{gathered}
$$

In the worst case,

$$
\Delta f_{\phi}=\alpha_{\phi} \operatorname{sgn}(s), \Delta f_{\theta}=\alpha_{\theta} \operatorname{sgn}(s)
$$

Therefore,

$$
\begin{gathered}
s_{1} \dot{s}_{1}=s_{1}\left[\alpha_{\phi} \operatorname{sgn}\left(s_{1}\right)-k_{1} \operatorname{sgn}\left(s_{1}\right)\right]=-s_{1} \eta_{1} \operatorname{sgn}\left(s_{1}\right)<0 \\
s_{2} \dot{s}_{2}=s_{2}\left[\alpha_{\theta} \operatorname{sgn}\left(s_{2}\right)-k_{2} \operatorname{sgn}\left(s_{2}\right)\right]=-s_{2} \eta_{2} \operatorname{sgn}\left(s_{2}\right)<0 \\
k_{1} \equiv \alpha_{\phi}+\eta_{1} \\
k_{2} \equiv \alpha_{\theta}+\eta_{2}
\end{gathered}
$$

\subsection{Road bank angle calculation}

Road bank angle calculation is a difficult subject of vehicle roll dynamics. It is difficult to estimate the road bank angle without GPS / GNS because the road bank angle and suspension displacement simultaneously contaminate the vehicle sensor signals. However, the three wheel tilting vehicle can identify the angle due to the suspension deflection due to the presence of the tilt angle sensor. Therefore, the road bank angle is simply calculated as the difference between the vehicle total roll angle and the tilt angle

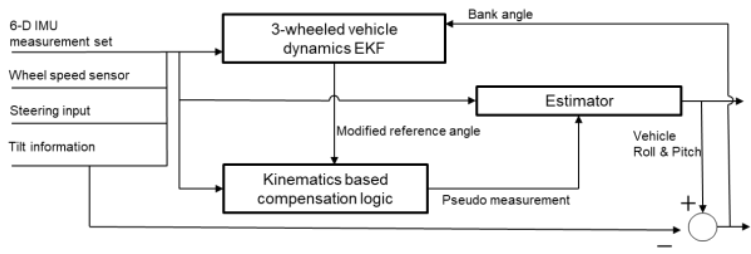

Figure 5. Road bank angle estimation schem

\section{Simulation Result}

To verify the proposed algorithm, simulation verification using Carsim and Matlab\&Simulink is conducted.
Various simulations are performed to test the robustness and stability of the algorithm. The table below enumerates various maneuver to test proposed algorithm.

Table 1. Test maneuver

\begin{tabular}{|c|c|c|c|}
\hline Road 1 & Velocity & Behavior \\
\hline Case 2 & $\begin{array}{c}\text { 15 deg ramp } \\
\text { bank }\end{array}$ & $80 \mathrm{kph}$ & Straight+DLC \\
\hline Case 3 & $\begin{array}{c}5 \text { deg ramp } \\
\text { bank }\end{array}$ & $80 \mathrm{kph}$ & $\begin{array}{c}\text { Normal } \\
\text { driving }\end{array}$ \\
\hline Case 4 & Flat & $20 \mathrm{kph}$ & $\begin{array}{c}\text { Sine wave } \\
\text { Steer }\end{array}$ \\
\hline Case 5 & Flat & $40 \mathrm{kph}$ & $\begin{array}{c}\text { Sine wave } \\
\text { steer with } \\
\text { tilting control }\end{array}$ \\
\hline
\end{tabular}

Case 1 maneuver is performed to verify the performance of the estimator for large amounts of lateral velocity and road bank angles. It is expected that there is a difference between the proposed estimator performance and previous estimates that ignore vehicle lateral velocity. On the other hand, Case 2 is conducted to evaluate the normal road performance of the proposed estimator. Case 3 is called "Fishhook", which is a test of vehicle stability testing. This extreme situation is simulated to evaluate the estimator stability and performance. It can be seen that the Case 4 and Case 5 simulation are not performed on the banked road. A sinusoidal steering input is entered to evaluate performance on the zero degree road bank angle.

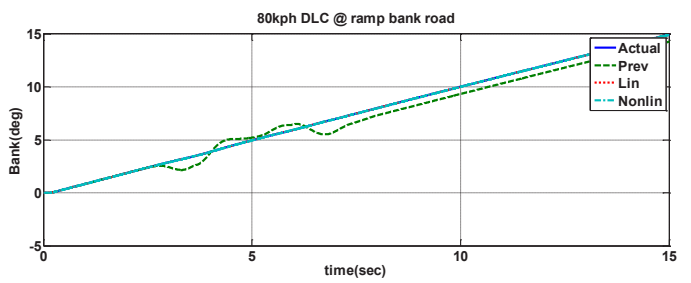

Figure 6. Case 1 Road bank angle

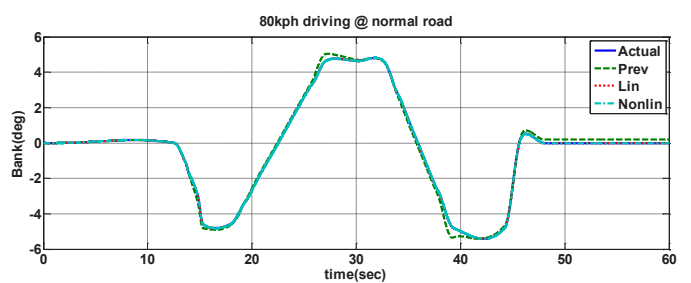

Figure 7. Case 2 Road bank angle

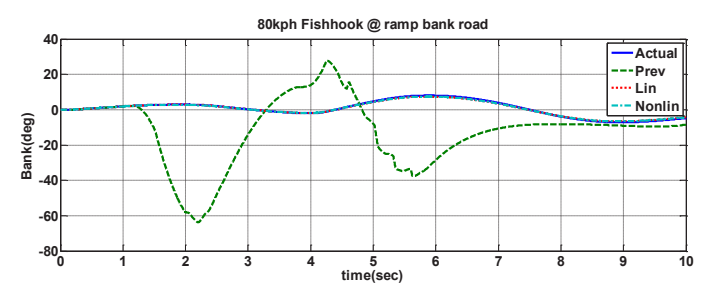

Figure 8. Case 3 Road bank angle 


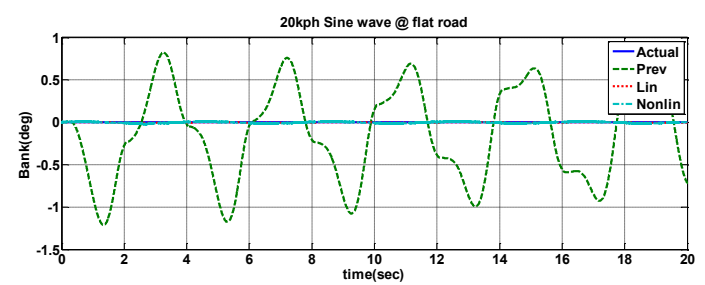

Figure 9. Case 4 Road bank angle

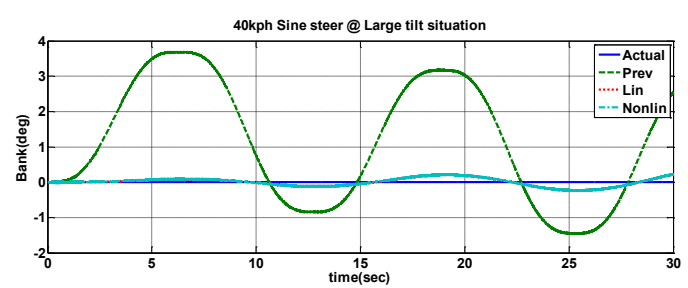

Figure 10. Case 5 Road bank angle

In this figures, "Actual" means simulation data. This data is considered actual vehicle test data. "Prev" means road bank angle estimation using only kinematic model based pseudo measurement such as those of previous result. "Lin" and "Nonlin" refer to the estimation result of linear and nonlinear estimators.

The figures above show the estimated road bank angle and the figures below show the estimated vehicle roll angle. From Case 1 to Case 3, there is few difference between road bank angle and vehicle roll angle. Therefore, the simulation results indicate that the vehicle suspension roll angle or vehicle tilt angle is small.



Figure 11. Case 1 Roll angle

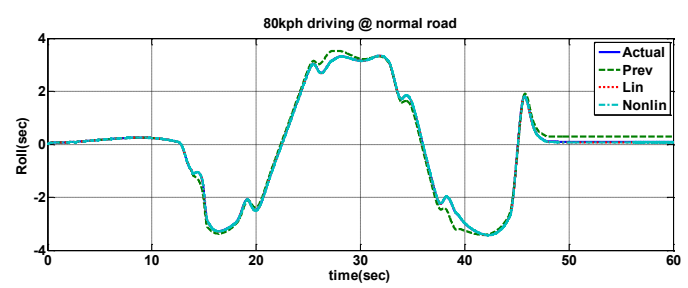

Figure 12. Case 2 Roll angle

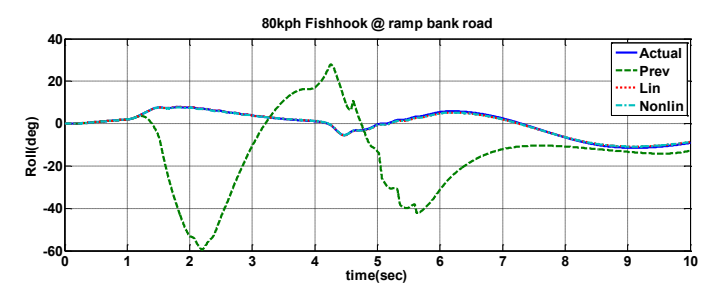

Figure 13. Case 3 Roll angle

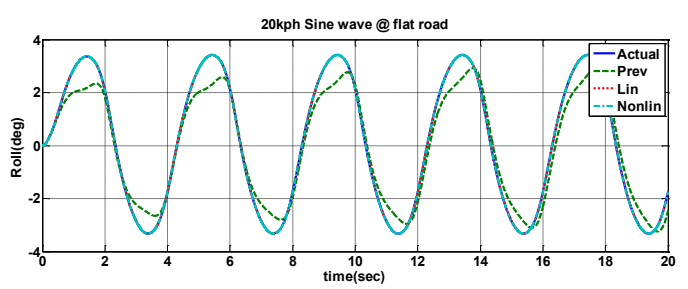

Figure 14. Case 4 Roll angle

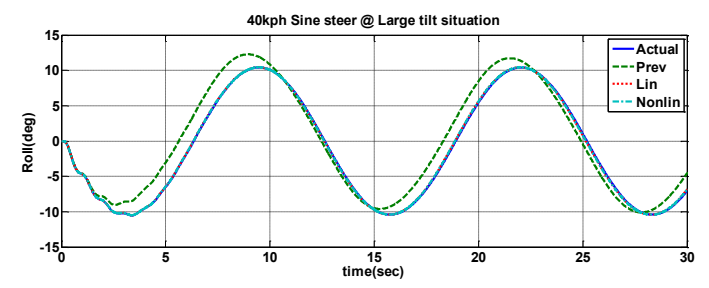

Figure 15. Case 5 Roll angle

Conversely, Case 4 and Case 5 roll and bank angle results show a significant difference between road bank angle and vehicle roll angle. Therefore, in this maneuver, the vehicle has a large inclination angle.

According to Case 2, in the normal driving situation, the proposed estimation method is slightly more accurate than the previous estimation. Since the tilt angle of the vehicle is small, the lateral dynamics are negligible. Therefore, the estimation results are almost unchanged. In the contrary, Case 1, Case 3, Case 4 and Case 5 show that the difference between the previous estimator result and proposed estimator results. Especially in case 4 and case 5 , the performance of the previous estimator was significantly reduced. On the other hand, from Case 1 to Case 5, the proposed evaluator provides precise results for various maneuvers.

\section{Conclusions}

In this paper, the estimator which is specialized the three wheel tilting vehicle is proposed. Using vehicle dynamic and kinematic model, the accurate pseudo measurement can be obtained in both steady and transient states. Unlike previous study, lateral dynamics are not neglected, and more accurate estimation results can be obtained from extreme maneuver. Also, unlike previous studies, nonlinear estimators is proposed for accuracy with large tilt angle maneuver. Other prior vehicle roll estimators designed for conventional four wheel vehicles are not accurate when the vehicle's tilt angle is large. However, this estimator is more accurate because the proposed estimator in this paper considers the dynamics of a threewheeled tilting vehicle. In conclusion, in this paper, the road angle estimator for three wheel tilting vehicle using 6D-IMU sensor is defined and verified. The main contribution of this paper is to propose a multi model estimator using steady state factor. This algorithm is suitable for three wheel tilting vehicle and verified using a custom ADAMS tilting vehicle. 


\section{Acknowledgement}

This work was supported in parts by the National Research Foundation of Korea grant funded by the Korea government (MSIP) (No.2017R1A2B4004116), the Ministry of Science and ICT, Korea, under the Information

Technology Research Center support program (IITP2017-2012-0-00628) supervised by the Institute for Information and communications Technology

Promotion, and the BK21 Plus Program.

\section{References}

1. Hibbard, Robin, and Dean Karnopp. "Twenty first century transportation system solutions-A new type of small, relatively tall and narrow active tilting commuter vehicle." Vehicle system dynamics $\mathbf{2 5 . 5}$ (1996):321-347.

2. Gohl, J., et al. "Development of a novel tiltcontrolled narrow commuter vehicle." (2006).

3. Robertson, James W., Jos Darling, and Andrew R. Plummer. "Combined steering-direct tilt control for the enhancement of narrow tilting vehicle stability." Proceedings of the Institution of Mechanical Engineers, Part D: Journal of Automobile Engineering 228.8 (2014): 847-862.

4. Piyabongkarn, D., T. Keviczky, and R. Rajamani. "Active direct tilt control for stability enhancement of a narrow commuter vehicle." International Journal of Automotive Technology 5.2 (2004): 77-88.

5. Gohl, J., et al. "Active roll mode control implementation on a narrow tilting vehicle." Vehicle system dynamics 42.5 (2004): 347-372.

6. Kidane, S., et al. "Road bank angle considerations in modeling and tilt stability controller design for narrow commuter vehicles." American Control Conference, 2006. IEEE, 2006.

7. Han, Kyoung Seok, et al. "Adaptive Scheme for the Real-Time Estimation of Tire-Road Friction Coefficient and Vehicle Velocity." IEEE/ASME Transactions on Mechatronics (2017).

8. Eric Tseng, H., Li Xu, and Davor Hrovat. "Estimation of land vehicle roll and pitch angles." Vehicle System Dynamics 45.5 (2007): 433443. 